\title{
PROBLEMS OF CHOOSING AN INTERMODULE INFORMATION INTERACTION PROTOCOL FOR MOBILE ROBOTS WITH MODUlar CONTROL SYSTEM ARCHITECTURE
}

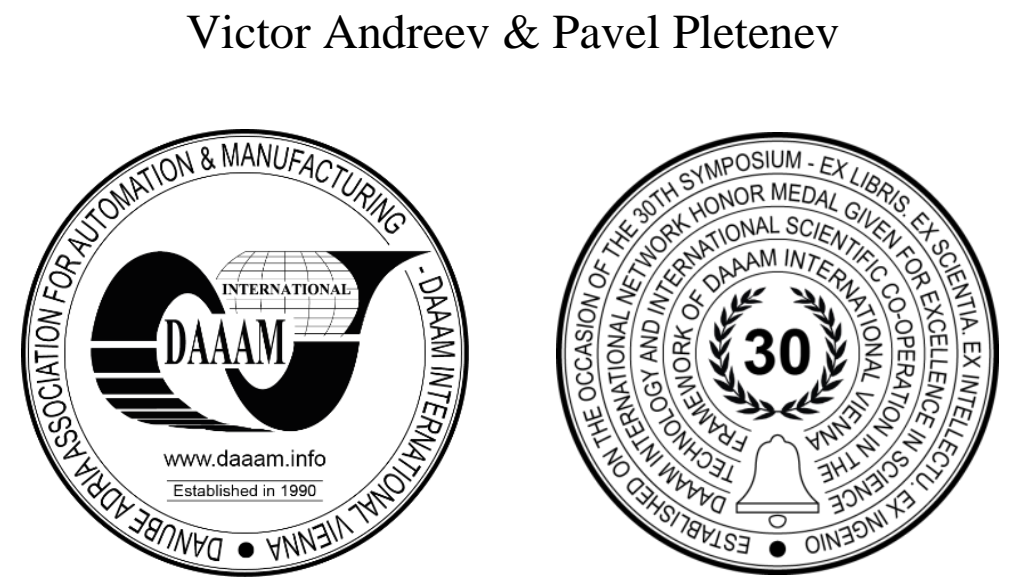

This Publication has to be referred as: Andreev, V[ictor] \& Pletenev, P[avel] (2021). Problems of Choosing an Intermodule Information Interaction Protocol for Mobile Robots with Modular Control System Architecture, Proceedings of the 32nd DAAAM International Symposium, pp.0151-0157, B. Katalinic (Ed.), Published by DAAAM International, ISBN 978-3-902734-33-4, ISSN 1726-9679, Vienna, Austria

DOI: $10.2507 / 32$ nd.daaam.proceedings.024

\begin{abstract}
In the modern world, there is an increasing need to combine the results of the development of various engineers, vendors and research groups to quickly create and efficiently operate robotic systems (RTS) for various purposes. The creation of common ways of interaction between parts of the software and hardware of mechatronic devices and robotic systems (modules) is one of the important tasks facing robotics as a science and a field of technology. The article proposes a criterion for assessing the applicability of existing protocols for creating methods of intermodule communication in RTS with a modular control system architecture. The criterion includes 12 requirements, the presence or absence of which determines the degree of suitability for the implementation of intermodular information interaction in the modular structure of a robotic system. As a result of the analysis of existing systems, the main sources of problems of organizing such interaction are identified and the applicability of the protocol chosen based on this criterion for the multilevel hierarchical (pyramidal) network topology of the RTS control system is justified. The article proposes a new concept of a generalized method of intermodule interaction implemented in the form of a specification.
\end{abstract}

Keywords: modular robot; communication interface; protocols; internet of things.

\section{Introduction}

When using robotic systems (RTS) in extreme scenarios, it is often impossible to predict the conditions in which this RTS will work; therefore, it is impossible to determine its functional composition in advance. There are times when the robot must be fully or partially functionally modified at the site of the upcoming work (or even during the work itself) that is, the robot must have the ability to perform operational reconfiguration. The works [1], [2], [3] brought attention to this problem. Control systems with a mono-computer do not allow for operational reconfiguration of the RTS; to ensure the reconfigurability of a mobile robot (MR) with a centralized control system (CS) in practice means creating a new robot with a given new functionality. As noted in [4], the solution to these problems is seen in the creation of a modular architecture of the control system of a mobile robot. 
Operational reconfiguration is a change in the configuration and/or technical characteristics of a mobile robot when adding new functional units or replacing them without the need to reconfigure the software (software) of the control system of the entire robot. The requirement of efficiency leads to the need for such an organization of the CS, in which it is automatically set to a new configuration - plug-and-play mode. Since such RTSs operate in difficult and dangerous conditions, the requirements for autonomy, and, consequently, for the speed of reaction to a changing situation in such RTSs are increasing. This entails ensuring the real-time operation of the RTS for all components of the robot.

This article is a continuation of the research [5], [6], [7] to develop the principles of creating a network infrastructure for modular mobile robots with a hierarchical pyramidal structure of the control system. This topic is part of a general new direction related to the creation of mobile robots based on modular architectures. Due to its novelty, this direction has not yet been clearly defined. Even though such systems consist of modules and are capable of reconfiguration, they do not belong to either homogeneous or heterogeneous robots. This is due to differences in the very concept of construction: in both homogeneous and heterogeneous robots, the implementation of reconfiguration is solved by changing the mechanical configuration - a kinematic scheme consisting of a limited set of modules, due to their rearrangement and mutual displacement. In an MR with a modular architecture, the target task is solved via the correct selection of functional nodes-modules and their automatic software integration into the robot's control system as a whole without any changes in the software aspects of both the control systems of the modules themselves and the general control system. Distributed computing is implemented in the modular architecture of the control system, which eliminates the problem of an infinite increase in the computing power of the control computer to provide real-time mode.

There are reasons to believe that the modular construction of the control system makes it possible to improve the functionality of a robot as a whole. This can be achieved by reducing energy consumption and heat dissipation of the entire control system due to the use of energy-efficient embedded systems - microcontrollers of low performance in the modules. The possibility of using such computing devices in modules is provided by reducing the complexity of algorithmic solutions of the functionality of a separate module. Also, an increase in the overall functionality of the RTS can be achieved through the division of engineering labor during the development of the robot - the designer of a separate module can find more effective and/or intelligent solutions than the designer of the robot as a whole, forced to deal with all design problems simultaneously. As soon as modules of sufficiently general purpose are created by different manufacturers for any of the fields of robotics, the use of such modules will be economically advantageous due to their mass production and competition between manufacturers. Therefore, it is necessary to create such means of intermodule interaction so that modules with the same functionality from different manufacturers are interchangeable.

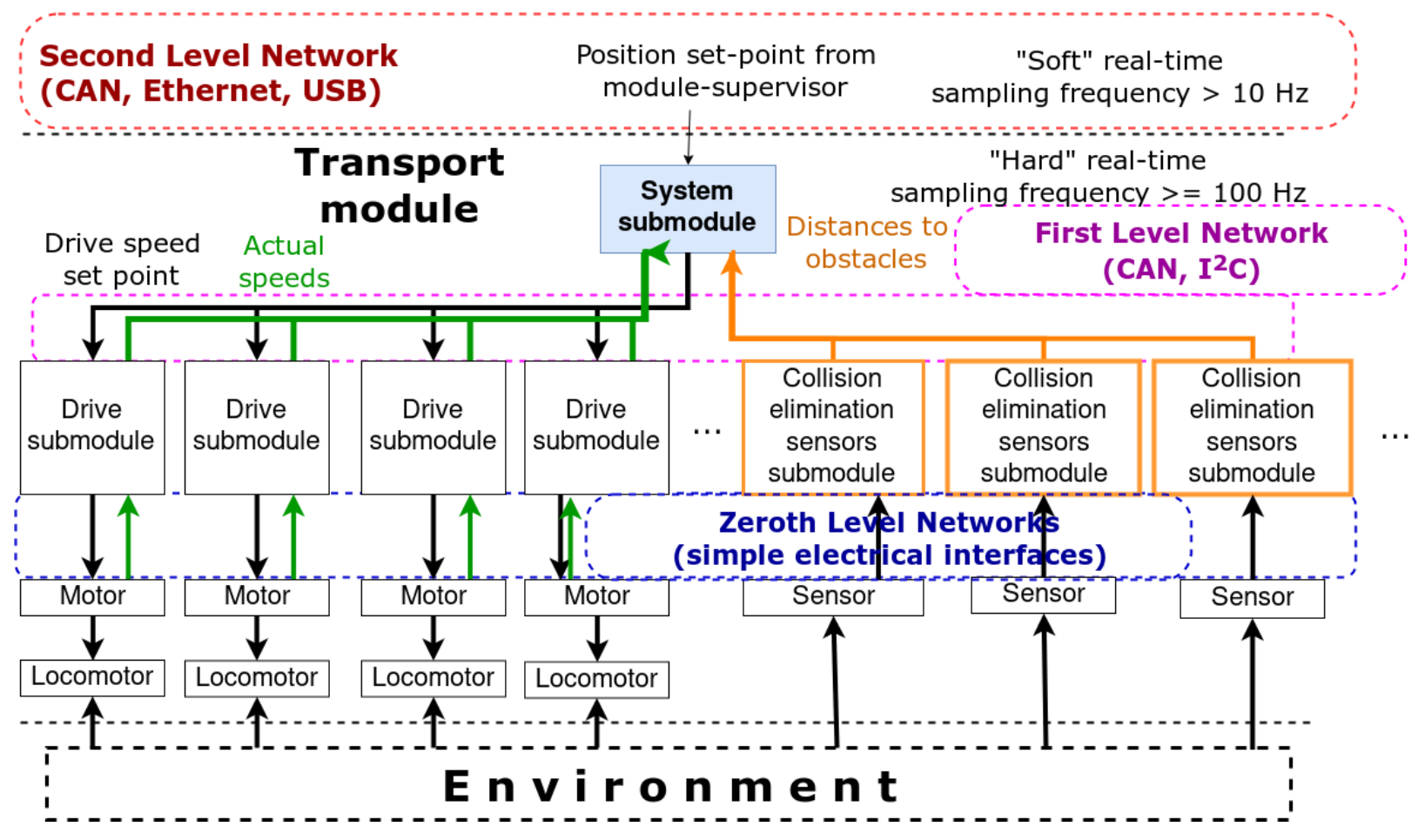

Fig. 1. Transport module's structure.

As a theoretical justification in [8], it was shown that the multilevel hierarchical (pyramidal) network topology of the MR control system can be represented as a multi-agent system in which "agent-modules" must interact with each other when performing their specific tasks. In a mobile robot with a modular architecture, considered in [6] and [7], interaction between different modules and submodules occurs at different levels of the hierarchy of agent-modules through different environments. 
Such an environment is made up of service software that implements various communication protocols at different levels of the OSI model [9]. At the same time, in an MR with a modular hierarchical CS structure, there may be many such interaction environments. In our previous works, at least two levels of subordination of interaction environments were identified - communication networks of the first and second levels (an example of such a structure of a transport module is shown in Figure 1). In these works, studies were conducted on the applicability of various transport protocols for the operation of a transport module in a modular robot. It has been shown that the CAN bus and the UDP/IP protocol from the Ethernet technology stack have sufficient speeds for use in modular robots. These two protocols are well-known and popular among software developers for different purposes, but they require the introduction of a data exchange discipline - that is, network protocols of a higher (in the sense of OSI) level - the application level. Such a protocol will be called the intermodule communication method (IMCM). IMCM can be built on one or more transport layer network protocols (CAN, UDP/IP, TCP/IP, etc.), which we will call "IMCM transports" for short.

The purpose of this work is to develop requirements for methods of intermodule communication of agentsmodules, on the basis of which to assess the applicability of existing protocols for creating IMCMs in the hierarchical structure of mobile robots with modular architecture, to identify their strengths and weaknesses.

\section{Requirements for the method of intermodule interaction}

Based on the results of previous studies [5], [6], [7] it is possible to formulate the following requirements for IMCM in the hierarchical structure of the control system of a modular mobile robot:

R1. IMCM's transports must ensure the data transfer speed, sufficient for the functioning of CS of modules in real time, and, if necessary, to provide deterministic message delivery.

R2. IMCM must be defined as an open standard or an open source specification.

R3. IMCM must have one or more portable (multi-platform), and preferably open implementations.

R4. It is desirable that IMCM can be implemented on top of different transports (see definition above).

R5. IMCM should be implementable on relatively simple (low performance) and cheap microcontrollers and occupy a minimum amount of both their program memory and RAM.

R6. IMCM must support the connection and disconnection of modules during operation (hotplug).

R7. Messages in IMCM must be typed. Message exchange in IMCM should be built to exclude the reception and transmission of corrupted or incorrect typed messages.

R8. IMCM transports should not have an explicit main node for data transmission, i.e. the communication network should ensure the creation of direct horizontal links.

R9. IMCM with transports that are able to provide the node with information about the topology of the connection of other nodes in the network should have priority.

R10. There should be a unified way of transmitting messages between different IMCM transports.

R11. As many levels as possible (in the sense of OSI) of IMCM transport protocols should be implemented at the hardware level in embedded systems (microcontrollers and embedded computers).

R12. The physical and/or channel layer of IMCM transports must be protected from interference and/or have error detection and correction mechanisms for transmitting information at module-specific distances.

The R1 and R12 requirements are necessary so that the modules can reliably maintain real-time operation even in the presence of external interference. It should be noted that the requirements of R2 and R3 are key since they form the requirement of interoperability (in the sense of the standard [10]). In case of non-compliance, the use of IMCM to combine modules from different manufacturers and different teams will be extremely difficult due to the lack of a unified approach to implementation. In order to achieve the stated goals of reducing energy consumption and minimizing cost, the requirements R5 and R11 have been introduced. The requirements R4 and R10 are necessary in order to be able to select the same IMCMs for different levels of the hierarchy. The R6 requirement is important for implementing operational reconfiguration. The R7 requirement is important for creating easy-to-understand and errorresistant IMCMs.

Since the modules in a modular robot must work in real time, a robot should not have a central point of failure of all systems - the R8 requirement is responsible for this. Since changing the structure of a modular robot (reconfiguration) changes various characteristics (its mass, moment of inertia, etc.), it is necessary to rebuild and adjust the control systems of robot modules. Therefore, in order for the mechanisms to work correctly during the automatic reconfiguration of modules and submodules at all levels, it is necessary to automatically update the knowledge of modules about the relative location of modules and submodules, their size, weight, and so on. Thus, IMCM must provide modules with information about their mutual topology, i.e. about where exactly the next module is connected or disconnected from - requirement R9. 
It should be noted that R8 and R9, in fact, contradict each other. Therefore, any choice of IMCM will be a compromise between the possibility of creating horizontal connections and obtaining information about the topology and connection points of nodes. For each of the IMCM under consideration, we will evaluate compliance with the corresponding requirement with the values of 1 (met) or 0 (not met) points. Then we add up the points obtained by IMCM to form an overall assessment of the applicability of the IMCM for mobile robots with a modular hierarchical control system architecture. The maximum score is 12 points (all requirements are met).

\section{Assessment of the requirements in various networks}

We will conduct a study of the use of the infrastructure of various intermodule communication methods for integration possibilities, the use of ready-made program blocks and for the organization of intermodule interaction. Preliminary analysis of publications [7], [11], [12], [13], [14], [15], [16], [17], [18], [19] has shown that in many works there is a "unifying" approach to intermodule interaction - it is proposed to combine several "transport" communication networks with one common protocol of intermodule interaction. "Transport" networks are understood here as communication networks whose details of implementation and operation are hidden from the programmer by a generalized application programming interface (API).

Such "unifying" is, for example, IMCM EmSBoT [11]. The authors propose to create a common abstraction from various operating systems, both conventional - Linux and Windows, and real-time, such as Chibi/RTOS. It is also proposed to abstract from the way messages are transmitted between modules. The speed of the transports offered by EmSBoT is sufficient for service robots - Ethernet and CAN are used, which is also implemented in our previous work [7] - the R1 requirement is met. Article [11], unfortunately, is the only source of information about EmSBoT, i.e. there is no open specification, as there is no available implementation of this technology - the requirements of R2 and R3 are not met. Because of this, it is difficult to assess the remaining requirements - we will consider them unfulfilled due to the lack of information provided by the authors. EmSBoT gets 1 point out of 12 .

IMCM R2P [12] is designed for various mechatronic and robotic devices, starting with toy robots and ending with mobile service platforms, unmanned aerial vehicles and ground vehicles. The authors of R2P do not provide unified mechanical components, but focus only on the electronic and software components necessary for robotic systems, as well as on tools for developing embedded robot software. For intermodule communication in R2P, the author's RTCAN real-time protocol is used [13]. RTCAN is primarily aimed at real-time communication, and not at fault tolerance: erroneous messages are only marked as damaged, and higher-level protocols (above RTCAN) are responsible for all retransmissions. The authors use only the CAN bus, which has sufficient bandwidth. Conclusion - requirement R1 is met, but requirement R4 is not met. The authors published the source code for intermodule interaction, but not the specification - the requirements of R3, R5 are met, and R2 is not. IMCM R2P is based only on the CAN network interaction with more complex modules based on Linux OS is carried out through the transmission of messages to IMCM ROS (about which below) - the requirements of R4 and R9 are not met, and the requirements of R8, R10, R12 are met. CAN can support disconnecting individual modules from the network, but it is not clear from the article whether there is support for dynamic connection and disconnection in the protocol - the R6 requirement is not met. All types of transmitted data are declared in common header files - the R7 requirement is met. Since the CAN network is used, which is usually implemented at the hardware level in microcontrollers, the R11 requirement is met. The total score for R2P is 10 out of 12 points.

The Robotic Operating System (ROS), its successor is ROS2 [14], its low-level implementations of H-ROS (Hardware ROS) [15] and microROS [16] are IMCMs and an ecosystem of code for robotic applications. In this article, we will not consider the old version - ROS1 due to its missing implementation on microcontrollers and H-ROS because further implementation of this project has stopped. The authors of ROS and ROS2 tend to use modern technologies that are standards for software development. Both versions use XML format that is difficult to read and write for configuration, and strictly Ethernet is used for network interaction. The first version of ROS used a synchronous connection based on its own protocol based on the TCP/IP protocol stack. The second version uses the DDS - Data Distribution Service technology, which is quite difficult to implement and understand by simple developers. Direct use of this technology on microcontrollers is impossible due to the huge (by the standards of microcontrollers) sizes of the resulting code. To cope with this complexity, the developers of DDS - Object Management Group - have developed an alternative specification - XRCE-DDS (eXtremely Resource Constrained Environment). This partially solves the problem, but there is still a need for a central node that performs arbitration of all messages and communication with the rest of the ROS2 network. Due to the protocol features, it is not possible to establish direct horizontal connections at the lower level. Also, the amount of code for XRCE-DDS to work is still quite large. The Ethernet network speeds are enough to work in soft real time - the R1 requirement is met. All protocols included in ROS2 and microROS are implemented in the form of specifications and there are different both open and closed DDS implementations (see [16]- the requirements of R2 and R3 are met. Theoretically, ROS2 can be built on top of any transports, since the specifics of a particular transport or its software implementation are hidden in separate ROS Middleware interface libraries, therefore, in theory, the R4 requirement is met. Due to the peculiarities of microROS, the requirements of R4, R5 and $\mathrm{R} 10$ are met. Thanks to DDS, ROS2 provides automatic detection and reconnection of elements - the R6 requirement is met. In ROS, all messages are typed - the R7 requirement is met. Due to XRCE-DDS, the requirements of R8 and R9 are not met. 
The R11 requirement is not met, since all microROS integration methods require the installation of additional peripheral devices - a rare microcontroller can be connected via Ethernet without an additional module. The Ethernet network is sufficiently protected from interference, but the UART interface, which is the basis for the interaction of the microROS module with the central broker module, is not resistant to interference, therefore, the requirement - R12 is not met. The total score for ROS2 and microROS is 8 out of 12 points.

Our approach, described in the previous work [7], to the creation of IMCM MODROB-1 is based on the design methods of ZeroMQ libraries - C4 and offers a common specification for all levels of interaction and its specification for implementation based on a different "transport" communication networks. Just like IMCM EmSBoT, MODROB-1 proposes abstraction from specific communication networks. As it was shown in the noted work, the speeds of the selected transports are sufficient for data transmission, therefore the requirement R1 is fulfilled. MODROB-1 was originally designed as an open specification and the authors had plans to open access to the source code of the implementations - then, from the authors' point of view, the requirements of R2 and R3 are met. MODROB-1 was originally designed to work only via the CAN network, but then it was expanded to work on other communication networks, such as Ethernet or UART - the requirements of R4, R11 and R12 are met.

The authors have an implementation of MODROB-1 for different microcontrollers and computers. The implementation of this specification requires a fairly small amount of RAM and program memory - the R5 requirement is met. IMCM MODROB supports hot-plugging and disconnecting individual modules - other modules "connected" to this module will simply stop receiving new variable values from the disconnected module. There is also no need for special configuration to "connect" the module, since all modules constantly publish their values and the introduction of a new module into the network occurs automatically. But the mechanisms of (de)centralized module detection and toplevel management mechanisms that should set a new configuration have not yet been described, i.e. there is no mechanism that should automatically enable it when a new module (submodule) is connected. Thus, the R6 requirement is not fully met. Messages in MODROB-1 have only 1 type - a single-length floating-point real number. Other types are not supported by the specification. This means that the R7 requirement has not been met. IMCM MODROB-1 assumes the use of one global bus on top of different transports - the requirement of R8 is met, and R9 is not. The message type is the same for all the subject communication networks - the requirements of R10 are met. IMCM MODROB-1 scores 9 out of 12 points.

IMCM UAVCAN (Uncomplicated Application-level Vehicular Communication And Networking) [18], [19] is a new open standard developing according to the open specification model (requirement R2) with open implementations in several programming languages for different embedded systems (requirements R3, R5). The author of the standard shows the simplicity of implementing UAVCAN on new platforms - only 1500 lines of code are enough [19]. The zero version of the standard assumed the use of only the CAN bus, but its further development to version 1 led to the inclusion in the "orbit" of implementations for other networks, such as UDP over Ethernet, UART and even the IEEE-802.15.4 wireless channel (requirements R1, R4, R11 and R12). This flexibility is achieved primarily due to the static typing of all messages (R7 requirement) and a single and extremely efficient binary encoding format for information to be transmitted through transports. The network was built initially to create direct horizontal links between modules without a central broker and the need to explicitly subscribe to certain publications (the requirements of R8 are met, and R9 is not). Also, the authors of the specification have released quite a lot of additional programs based on this specification. For example, this specification does not assume dynamic addresses for modules, but with the help of additional software, they can also be implemented. Also, additional software allows you to implement dynamic reprogramming of embedded systems (requirement R6). Since the binary interface is the same for all modules, it is possible to use any of the transports and transmit messages between networks (R10 requirements). The UAVCAN framework scores 11 out of 12 points.

\begin{tabular}{|c|c|c|c|c|c|}
\hline Req. & EmSBoT & R2P & ROS2 & MODROB-1 & UAVCAN \\
\hline R1 & $\mathrm{V}$ & $\mathrm{V}$ & $\mathrm{V}$ & $\mathrm{V}$ & $\mathrm{V}$ \\
\hline $\mathrm{R} 2$ & $X$ & $\mathrm{X}$ & $\mathrm{V}$ & $\mathrm{V}$ & $\mathrm{V}$ \\
\hline R3 & $\mathrm{X}$ & $\mathrm{V}$ & $\mathrm{V}$ & $\mathrm{V}$ & $\mathrm{V}$ \\
\hline $\mathrm{R} 4$ & $\mathrm{X}$ & $X$ & $\mathrm{~V}$ & $\mathrm{~V}$ & $\mathrm{~V}$ \\
\hline $\mathrm{R} 5$ & $\mathrm{X}$ & $\mathrm{V}$ & $\mathrm{V}$ & $\mathrm{V}$ & $\mathrm{V}$ \\
\hline R6 & $\mathrm{X}$ & $\mathrm{X}$ & $\mathrm{V}$ & $\bar{X}$ & $\mathrm{~V}$ \\
\hline R7 & $\mathrm{X}$ & $\mathrm{V}$ & $\mathrm{V}$ & $\mathrm{X}$ & $\mathrm{V}$ \\
\hline R8 & $X$ & $\mathrm{~V}$ & $\mathrm{X}$ & $\mathrm{V}$ & $\mathrm{V}$ \\
\hline R9 & $X$ & $\mathrm{X}$ & $X$ & $\mathrm{X}$ & $\mathrm{X}$ \\
\hline R10 & $X$ & $\mathrm{~V}$ & $\mathrm{~V}$ & $\mathrm{~V}$ & $\mathrm{~V}$ \\
\hline R11 & $X$ & $\mathrm{~V}$ & $\mathrm{X}$ & $\mathrm{V}$ & $\mathrm{V}$ \\
\hline R12 & $X$ & $\mathrm{~V}$ & $\mathrm{X}$ & $\mathrm{V}$ & $\mathrm{V}$ \\
\hline Sum & 1 & 8 & 8 & 9 & 11 \\
\hline
\end{tabular}

Table 1. Comparison of various MMK (V - the requirement is fulfilled, $\mathrm{X}$ - the requirement is not fulfilled) 
The results of the study are summarized in Table 1 . The letter V indicates fulfilled requirements, $\mathrm{X}-$ unfulfilled. As can be seen from the table, the most suitable for implementing the method of intermodule interaction are MODROB-1 and UAVCAN. These methods most fully implement the necessary requirements, except for the R9 requirement for determining the topology.

\section{Conclusion}

Our earlier research aimed at developing a protocol for intermodule interaction in the network structure of the control system of mobile robots with modular architecture was only partially successful. Nevertheless, these studies made it possible to develop requirements for such protocols and analyze existing protocols developed by other authors in order to assess the possibility of their use in intermodule communication methods (IMCM) in real-time systems. The analysis given in this paper showed that the UAVCAN protocol meets the listed requirements the most and differs from our developments (IMCM MODROB-1) in that it is already supported by the community of developers of unmanned aerial vehicles (UAVs). Thus, unlike IMCM MODROB-1, this protocol has a great prospect for use. There is not as much ready-made software written for UAVCAN as for ROS/ROS2, but proposals have already been made in the developer community to replace the DDS networking layer inside ROS2 with UAVCAN. Also, the software of a "bridge" between ROS and UAVCAN has already been written, which will allow reusing many of the developments in the field of software from ROS. However, its direct use in our hierarchical architecture of the control system of mobile modular robots is impossible. Although the UAV is also a mobile robot, its operating conditions and functional purpose are significantly different. In this regard, it is necessary to develop add-ons for the UAVCAN protocol - unified protocols for different types of modules and submodules and a unified set of software libraries designed to develop their software aspects. It is also necessary to experimentally test the throughput of intermodule communication channels with different protocols. It should also be checked whether UAVCAN is suitable for use both for basic control on microcontrollers and for more complex interaction at higher levels of the hierarchy, since hard or soft real-time mode is required at different levels of the hierarchy.

We believe that our further actions should be aimed at an experimental study of the use of the UAVCAN protocol in the communication network of a modular robot with a multi-level (hierarchical) control system architecture. It is also necessary to investigate the applicability of this protocol for the implementation of dynamic reprogramming of computing devices of control systems of modules and submodules.

\section{Acknowledgments}

Research is supported by the Russian Foundation for Basic Research: Grant 19-07-00892a.

\section{References}

[1] Babich, A.V.; Baranov, A.G.; Kalabin, I.V. \& others (1982). Industrial robotics[Промышленная робототехника], Mashinostroenie[Машиностроение], Moscow, 1982.

[2] Vorobyov E.I.; Kozyrev, Yu.G. \& Tsarenko, V.I. (1988) Industrial robots of aggregate-modular type [Промышленные роботы агрегатно-модульного типа], Mashinostroenie[Машиностроение], ISBN: 5217-00166-6, Moscow.

[3] Lopota A.V. \& Yurevich E.I. (2013) Stages and prospects of development of the modular principle of building robotic systems [Этапы и перспективы развития модульного принципа построения робототехнических систем], Scientific and technical bulletin of SPbSPU [Научно-технические ведомости СПбГПУ], Vol 12013 Computer science. Telecommunications. Management.[Информатика. Телекоммуникации. Управление.], pp. 98 - 103, ISSN 1994-2354

[4] Yurevich E.I. (2003) Robots of the Central Research Institute of RTK at the Chernobyl Nuclear Power Plant and the development of extreme robotics [Роботы ЦНИИ РТК на Чернобыльской АЭС и развитие экстремальной робототехники], Polytechnic University Publishing House [Издательство Политехнического университета], ISBN 5-7422-0481-7, Saint Petersburg

[5] Andreev, V. \& Pletenev, P. (2017) Organizing Intermodular Communication for Heterogeneous Modular Mobile Robot. pp. 0474-0480. https://doi.org/10.2507/28th.daaam.proceedings.066

[6] Andreev, V.P. \& Pletenev, P. (2019) Organizing Network Interaction in Modular Robot with Multilevel Control System, in: Proceedings of the 30th DAAAM International Symposium, DAAAM International, Vienna, Austria, pp. 0485-0492. https://doi.org/10.2507/30th.daaam.proceedings.065

[7] Andreev, V.; Pletenev, P. \& Eprikov, S., (2020). Development of a Communication Protocol Specification for The Modrob-1 Modular Mobile Robot. pp. 0244-0253. https://doi.org/10.2507/31st.daaam.proceedings.034

[8] Andreev V. (2019). Control System Mobile Robots with Modular Architecture as a Multi-Agent System with a Hierarchical Topology, Proceedings of the 30th DAAAM International Symposium, B. Katalinic (Ed.), Published by DAAAM International, ISBN 978-3-902734-22-8, ISSN 1726-9679, Vienna, Austria, 2019, pp.0010-0019

[9] GOST R ISO/MEK 7498-1-99. — «Information technology. Open systems interconnection. Basic reference model. Part 1. The basic model». — OKS: 35.100.70. — In use since 01.01.2000. — 62c. 
[10] ISO Central Secretary, (2021). Robotics - Modularity for service robots - Part 1: General requirements (Standard No. ISO/IEC TR 22166-1:2021). International Organization for Standardization, Geneva, CH.

[11] Peng, L.; Guan, F.; Perneel, L.; Fayyad-Kazan, H. \& Timmerman M. (2016) EmSBoT: A lightweight modular software framework for networked robotic systems. 2016 3rd International Conference on Advances in Computational Tools for Engineering Applications (ACTEA), Beirut, 2016, pp.216-221. doi: 10.1109/ACTEA.2016.7560142.

[12] Bonarini, A.; Matteucci, M.; Migliavacca, M .\& Rizzi, D. (2014). R2P: An open source hardware and software modular approach to robot prototyping. Robotics and Autonomous Systems. - 2014. - No.62. - pp.1073-1084.

[13] Migliavacca, M.; Bonarini, A. \& Matteucci, M., (2013). RTCAN - A Real-time CAN-bus Protocol for Robotic Applications, in: ICINCO. https://doi.org/10.5220/0004484303530360

[14] Thomas, D.; Woodall, W. \& Fernandez, E., 2014. Next-generation ROS: Building on DDS, in: ROSCon Chicago 2014. Open Robotics, Mountain View, CA. https://doi.org/10.36288/ROSCon2014-900183

[15] Mayoral, V.; Hernandez, A.; Kojcev, R.; Muguruza, I. \& others. (2017). The shift in the robotics paradigm - the Hardware Robot Operating System (H-ROS); an infrastructure to create interoperable robot components, NASA/ESA Conference on Adaptive Hardware and Systems (AHS), Pasadena, CA. - 2017. - pp.229-236.

[16] micro-ROS URL https://micro.ros.org/ (accessed 29.09.21).

[17] Kronauer, T., Pohlmann, J., Matthe, M., Smejkal, T., Fettweis, G., 2021. Latency Analysis of ROS2 Multi-Node Systems. arXiv:2101.02074 [cs].

[18] Kirienko, P.; Dixon, S. \& others (2014) UAVCAN: an open lightweight protocol for reliable intravehicular communication in aerospace and robotic applications over CAN bus, Ethernet, and other robust transports. Available from: https://uavcan.org/specification/UAVCAN_Specification_v1.0-beta.pdf (accessed 29.09. 21).

[19] Kirienko, P. (2019). Publisher/subscriber for distributed fault-tolerant real-time onboard systems in 1500 lines of code [Издатель/подписчик для распределённых отказоустойчивых бортовых систем реального времени в 1500 строк кода] URL https://habr.com/ru/post/512570/ (accessed 29.09.21) 